Benson, Thomas S., and Christina A. Boyes

\title{
Health and Well-being in Graduate School: Preventing Burnout
}

\section{What do you need to know about health and well-being in graduate school?}

\section{The Problem}

Health and well-being are essential for political science graduate students who are aspiring to produce high-quality work, engage effectively in the profession, maintain healthy relationships and worklife balance, and - most importantly - be physically and mentally well. Unfortunately, Western society is experiencing a "mental health crisis" and "epidemic of work stress" (Väänänen and Varje 2019, 37). The discipline of political science is no exception to this (Lau and Pretorius 2019, 38). Overwork culture drives many graduate students to sacrifice health and well-being due to high workloads, isolation, competition for research funding, pressure to publish, career and financial insecurity, and lack of a support system (Lau and Pretorius 2019, 39). Additional duties include the pursuit of teaching excellence, community engagement, and managing familial expectations.

Collectively, these duties have led to many graduate students in the discipline to adopt their professional status as a form of identity that becomes all-consuming. This identity establishes an "exaggerated sense of duty," in which graduate students only feel they can relax once their duties have been completed but the "desk never becomes empty" and housework is seemingly never-ending (PirkerBinder 2016, 108). By failing to conform to the norms constructed by the discipline, students subsequently feel as though their identity is being threatened (Dick 2019, 163-164). Those who experience their identity being threatened may encounter imposter syndrome - feeling as though they are inadequate and cannot achieve the incessant demands and high expectations placed upon them because they believe themselves to be frauds (Dick 2019, 171). Ultimately, then, political science graduate students can feel deflated, experiencing burnout, chronic stress, depressive symptoms, fatigue, irritability, suicidal ideation, and anxiety (Väänänen and Varje 2019, 38; Lau and Pretorius 2019, 40) - all of which can undermine health and well-being.

Stress from professional and personal demands and expectations attributed to the norms of the discipline can be understood as the "perceptions or feelings a person experiences when a particular environment taxes or exceeds the person's available personal or social coping resources" (Lau 2019, 48). Such stress is often triggered by "uncontrollable aversive challenge[s]" (Fink 2016, 4), including anxiety regarding conference presentations or teaching undergraduate students, conducting fieldwork, or sharing critical thoughts regarding political science literature or data in a classroom, among other things. These stressful and anxiety-inducing situations can have physiological and cardiovascular consequences and are each influenced by a student's "perception of their ability to cope with the stressor," thereby suggesting that stress is subjective (Fink 2016, 5; Väänänen and Varje 2019, 39, 52). Stress and burnout also manifest as tiredness, muscle tension, and headaches.

The experience of health challenges during graduate study is normal. This does not demean the experiences of graduate students but acknowledges them. Experiencing these issues does not make you unfit to be a graduate student. Generally, graduate students are more likely to experience depression and anxiety than the general population (Evans et al. 2018, 282), and some have reported low quality of life, 
Benson, Thomas S., and Christina A. Boyes

poor health, or feeling disconnected, depressed and overwhelmed by their workload (Yusuf et al. 2020, 468). There is a "mental health crisis" in academia (Lau and Pretorius 2019, 38). One study found that $15.8 \%$ of graduate students in political science at seven U.S. universities expressed thoughts of suicide in two weeks prior to the survey, $30 \%$ were depressed, and about $32 \%$ were experiencing anxiety (Almasri, Read, and Vendeweerdt 2021). Given these challenges, we provide some potential solutions in the following subsection with greater emphasis on solutions in the final section.

\section{Potential Solutions}

Do not suffer in silence. Your adviser and graduate director should support your well-being, but they will not know you are struggling if you remain silent (Lau and Pretorius 2019, 39). Most faculty are supportive and open; speak to them about well-being, burnout, stress, anxiety, and imposter syndrome. It is likely that they - who were once graduate students - experienced the same issues. Similarly, you should speak openly with your peers - they are likely experiencing the same issues (Lau 2019, 49). Together, you can amplify concerns to your department or administration and address them as a collective, should there be lackluster resources available on campus (e.g., therapy, counseling, mental well-being services). If you have an assistantship, you likely have health insurance provided by your employer - if this is the case and your institution does not provide adequate support, consider off-campus resources.

Being a graduate student in political science is job training in teaching, research and studying. Establish a work-life balance at the outset of your graduate program to keep your health and well-being on track. Maintaining work-life balance will likely have a positive impact on your quality of life (Yusuf et al. 2020, 470-471). As part of this, try different methods of note-taking, tracking deadlines, setting shortand long-term goals to keep motivated, building a support network (faculty, peers, staff, external mentors), identifying helpful resources, exercising, getting adequate sleep, going outside to get fresh air and sunlight, maintaining a healthy diet, staying hydrated, and being efficient with your time. However, be wary of advocates that call only for micro-level solutions as the culture of overwork is systemic, thereby warranting broader change in political science departments and academia as a whole (Väänänen and Varje 2019, 51).

\section{Why does health and well-being matter in graduate school?}

The first step to developing an understanding of your health and well-being is to get tested and treated if you suspect you are experiencing any health challenge, be it chronic (e.g., chronic stress, back pain) or temporary (e.g., panic attack, sore throat). Identify any particular issues (e.g., OCD, ADHD, anxiety, depression, allergies, visionary challenges, chronic illness), so you can address them and prevent them from interfering with your quality of life. Remember: mental health is health too, and healthcare is confidential in many countries, including the U.S. Graduate students should not be concerned about stigma resulting from diagnoses, especially international students who may come from cultures that stigmatize mental or physical health issues. Recognize that you have basic needs as a human being and develop coping resources to maintain your wellbeing (Muniroh 2019, 155).

Poor health can adversely affect your capacity to work and study. This can further reinforce stress that you may already be experiencing (Lau and Pretorius 2019, 39), provoking a cyclical process of 
chronic stress (Lau 2019, 48) and sleep deprivation that can lead to "daytime sleepiness and cognitive impairments." Graduate students who reduce sleep to maximize study and work time can experience negative impacts on their success and quality of life (Lau 2019, 54; Calvo and Gutiérrez-García 2016, 143). However, not all stress is bad. Acute short-term stress (e.g., minutes or hours) can have some beneficial effects, such as memory consolidation (Calvo and Gutiérrez-García 2016, 142).

Burnout, conversely, is especially bad. Burnout has been described as being "comparable to a house that burns and consumes itself from the inside, without always leaving visible traces on the outside" (Kirouac 2019, 178). It has also been defined as a "transient mental illness" and a "state of fatigue or frustration" that stems from failure to meet your expectations (Kirouac 2019, 181-182). Symptoms of burnout include loss of positivity, decrease in the feeling of personal accomplishment, emotional exhaustion, and reduced productivity (Kirouac 2019, 182). Students who excessively compete with their peers may compound these issues by exacerbating imposter syndrome (Lau 2019, 50).

Health challenges differ across groups. Some graduate students manage their own health and that of others (e.g., care-taking responsibilities for partners, parents, and dependents). Issues vary across academic years too, with some third- and fourth-year students reporting comparatively higher levels of stress than students in their initial years (Rico and Bunge 2021, 180). This could be because of the increased focus on dissertation work, fieldwork, and job applications (on top of regular duties, such as teaching and research) compared to the class workload and research and teaching obligations for graduate students in their initial years (Rico and Bunge 2021, 180).

Due to poor cultural understanding by some healthcare providers, BIPOC and international student populations may experience misdiagnoses - this may also be problematic for international students who do not have English as a first language - that subsequently disincentivize future use of mental and physical health services (APA 2017a, 2). For example, many Asians are less likely to use mental health services compared to race and ethnic groups (APA 2017a, 2). Some populations are more likely to experience persistent depression, post-traumatic stress disorder and alcohol dependence (APA 2017a, 1) which can be exacerbated by avoiding treatment.

Women are more likely than men to experience PTSD, anxiety, suicide, depression, and eating disorders (APA 2017c, 1-2). Barriers to women's treatment include stigma, inability to find time (e.g. time off work, childcare, transportation) to seek help, and lack of insurance (APA 2017c, 2). Additionally, LGBTQIA+ individuals are more than twice as likely as their heterosexual counterparts to experience a mental disorder in their lifetime, and 2.5 times more likely to experience depression, anxiety, or substance misuse (APA 2017b, 2). Many report experiencing stigma and discrimination when using health services, which leads to delayed or foregoing necessary healthcare (APA 2017b, 3).

\section{What can you do about your health and well-being?}

\section{Prevent Health Issues}

Graduate school is a time when pressure is high and competition between cohorts and colleagues can be daunting. In the first year, anxiety and imposter syndrome typically complicate the scene. Later, qualifying papers, comprehensive exams, conducting fieldwork, and navigating post-comprehensive exam 
expectations each present their own challenges. While each major milestone in a graduate school program is challenging in different ways, being prepared can help prevent physical and mental health struggles from interfering with your performance.

One of the first steps to staying healthy in graduate school is preventing health problems and getting yourself tested if you suspect any problems. In your first year, identify existing resources and befriend those who are in the same position as you and commiserate with each other. However, do not let competition drive your interactions. There is no reason to compare yourself with others. Instead, recognize your own limits and focus on doing better every day. Focusing on the performance of your peers will likely only lead to you believing you are unfit to be a political science graduate student.

If you need to access support resources, on-campus facilities are likely to include stress, work-life balance, and productivity workshops, nutritionists, counseling, therapy, gyms, pools, and in some cases, short-term programs that feature emotional support dogs at the end of semester or recreational opportunities to destress. Take advantage of these resources!

Most faculty have faced their own health struggles and will view self-care as a sensible choice. However, not every department is supportive of student health struggles. If you are uncomfortable looking within your department for support, consider talking with faculty in other departments with whom you share good rapport. Additionally, within the field, American Political Science Association offers many resources online and conferences provide good opportunities for networking and identifying additional resources.

If your campus does not offer many health resources or recreational opportunities, does the surrounding community? Can you hike, bike, or run? Are there local health clubs available? Integrating into the community can also help you escape the stress of graduate school. Sometimes, being surrounded by others facing similar struggles can be beneficial, but it can also amplify anxiety around milestones like comprehensive exams. Not everyone is comfortable with public activities that are health-focused, which is fine. If you prefer not to utilize local resources, there are several options online too. Bloggers such as Mirya Holman ${ }^{1}$ and Raul Pacheco-Vega ${ }^{2}$ are accomplished political scientists who work to pave the way for future and early career academics by sharing tips on writing, reading, and organizing notes, as well as providing inspiration, motivation, and community for their readers. You can also find online support communities on Twitter, Facebook, and Instagram.

There are many independent activities you can do to reduce your risk of health issues. Several apps can help you cultivate a healthy routine for better academic productivity, such as: Calm; Headspace; iStudiez (primarily for undergraduates but still useful); Focus To-Do; Google Tasks and Calendar; Habit; FLIP; and Flora - Green Focus. Beyond apps, productivity methods such as Pomodoro, Eat the Frog, or the 3 Box Method/Ivy Lee can be useful. Many graduate students find using daily planners, to-do lists, and block scheduling helpful. The key to productivity is to find something that works for you that you use consistently.

\footnotetext{
${ }^{1}$ See https://miryaholman.substack.com/

${ }^{2}$ See http://www.raulpacheco.org/blog/
} 


\section{If You Are Already Struggling}

Much of what applies for prevention applies when you are already struggling. The preceding section offers some ideas that may be useful. There are additional ways to address existing health problems, which we focus on in this section.

Before making any changes to your routine, remember that sweeping changes may work for a bit but can be too difficult to maintain. Little, sustainable changes may be easier to continue in the long term. Establishing accountability mechanisms and letting your support network know you are struggling can help. If you are new to your program, make sure that your Director of Graduate Studies (DGS) and your advisor (or a professor you feel safe confiding in) are aware of your struggles. Also, keep relevant administrative personnel in the loop to ensure that you do not miss key deadlines.

Physical and mental health are impacted by nutrition, yet food insecurity is often an issue for graduate students (Coffino et al. 2021). If you are food insecure, look for on- and off-campus food pantries and check to see if your university has on-campus dining or food hall cards for student financial aid recipients. Healthy eating, regardless of how apparently healthy you seem, can be beneficial, helping resolve existing health issues and preventing further health problems.

If you are dealing with stress, anxiety, depression, or impostor syndrome, you are not alone. Health issues are prevalent among graduate students. Sometimes, sharing your struggles with your peers alleviates issues like impostor syndrome by creating a sense of belonging (Lau 2019, 53). Everyone struggles. Hearing you are not facing impostor syndrome alone can be cathartic. Many students also benefit from actively expressing and thinking through their problems, either orally, visually, or in written form. These creative processes may help you work through problems that can be resolved without external intervention (Muniroh 2019, 116).

Regularly practiced mindfulness (potentially with the assistance of apps (e.g., Wysa, Headspace, or Smiling Mind), self-compassion, support networks, counseling, regular exercise, good sleep habits, and a balanced diet can improve intrapersonal wellbeing (Lau and Pretorius 2019, 42; Lau 2019, 52). Having a life outside of the department is also a good way to improve well-being and decrease stress and anxiety. External friendship networks can remove you from the stress of graduate school and help you maintain a work-life balance. Finally, by integrating with your community and spending more time with friends and family or people who share your recreational interests, you can also tackle impostor syndrome through shared experiences of belonging (Lau 2019, 53).

\section{References}

Almasri, Nasir., Blair Read., and Clara Vandeweerdt. 2021. "Mental Health and the PhD: Insights and Implications for Political Science.” PS: Political Science \& Politics: 1-7. doi: https://doi.org/10.1017/S1049096521001396.

American Psychiatric Association (APA). 2017a. "Mental Health Disparities: Diverse Populations." APA [website], December 12, 2021. https://www.psychiatry.org/psychiatrists/culturalcompetency/education/mental-health-facts. 
Benson, Thomas S., and Christina A. Boyes

American Psychiatric Association (APA). 2017b. "Mental Health Facts for Lesbian, Gay, Bisexual, Transgender, Queer/Questioning (LGBTQ).” APA [website], December 12, 2021.

https://www.psychiatry.org/psychiatrists/cultural-competency/education/mental-health-facts.

American Psychiatric Association (APA). 2017c. "Mental Health Facts for Women." APA [website], December 12, 2021. https://www.psychiatry.org/psychiatrists/cultural-competency/education/mentalhealth-facts.

Calvo, Manuel, G., and Aída Gutiérrez-García. 2016. "Cognition and Stress.” In Stress: Concepts, Cognition, Emotion, and Behavior: Handbook of Stress Series, ed. George Fink, 139-144. Elsevier: Academic Press.

Coffino, Jaime A., Samantha P. Spoor., Rae D. Drach., and Julia M. Hormones. 2021. "Food Insecurity Among Graduate Students: Prevalence and Association with Depression, Anxiety and Stress." Public Health Nutrition 24(7): 1889-1894. doi: https://doi.org/10.1017/S1368980020002001.

Dick, Penny. 2019. "Understanding Stress as a Form of Institutional Maintenance and Disruption Work." In Stress and Suffering at Work: The Role of Culture and Society, ed. Marc Loriol, 155-173. Cham, Switzerland: Palgrave Macmillan.

Evans, Teresa M., Lindsay Bira., Jazmin B. Gastelum., L. T. Weiss., and Nathan L. Vanderford. 2018. "Evidence for a Mental Health Crisis in Graduate Education." Nature Biotechnology 36: 282-284. doi: https://doi.org/10.1038/nbt.4089.

Fink, George. 2016. "Stress, Definitions, Mechanisms, and Effects Outlined: Lessons from Anxiety." In Stress: Concepts, Cognition, Emotion, and Behavior: Handbook of Stress Series, ed. George Fink, 3-11. Elsevier: Academic Press.

Kirouac, Laurie. 2019. "Burnout in Quebec. Behind Psychological Suffering, Shifting in Social Representation and Relation to Work." In Stress and Suffering at Work: The Role of Culture and Society, ed. Marc Loriol, 177-190. Cham, Switzerland: Palgrave Macmillan.

Muniroh, Siti. 2019. "Maintaining Emotional Wellbeing for Doctoral Students: Indonesian Students' Mechanism of Thinking Out Loud." In Wellbeing in Doctoral Education: Insights and Guidance from the Student Experience, ed. Lynette Pretorious, 113-126. Singapore: Springer.

Lau, Ricky W. K. 2019. "You Are Not Your PhD: Managing Stress During Doctoral Candidature.” In Wellbeing in Doctoral Education: Insights and Guidance from the Student Experience, ed. Lynette Pretorious, 47-58. Singapore: Springer.

Lau, Ricky W. K., and Lynette Pretorius. 2019. "Intrapersonal Wellbeing and the Academic Mental Health Crisis." In Wellbeing in Doctoral Education: Insights and Guidance from the Student Experience, ed. Lynette Pretorious, 37-45. Singapore: Springer. 
Benson, Thomas S., and Christina A. Boyes

Pirker-Binder, Ingrid. 2016. "The Working Human-The Exhausted Human.” In Mindful Prevention of Burnout in Workplace Health Management, ed. Ingrid Pirker-Binder, 107-123. Cham, Switzerland: Springer.

Rico, Yvette., and Eduardo L. Bunge. 2021. "Stress and Burnout in Psychology Doctoral Students." Psychology, Health \& Medicine 26(2): 177-183. doi: 10.1080/13548506.2020.1842471.

Väänänen, Ari., and Pekka Varje. 2019. "Epidemiological Transition and the Emergence of Mental Discomfort." In Stress and Suffering at Work: The Role of Culture and Society, ed. Marc Loriol, 37-54. Cham, Switzerland: Palgrave Macmillan.

Yusuf, Juita-Elena (Wie)., Marina Saitgalina., and David W. Chapman. 2020. "Work-life Balance and Well-being of Graduate Students." Journal of Public Affairs Education 26(4): 458-483. doi: https://doi.org/10.1080/15236803.2020.1771990. 